\title{
A Geometric Test for the Analysis of Contingency Tables
}

\author{
J. Quigley \& K.J. Wilson \\ Department of Management Science, University of Strathclyde, UK
}

\begin{abstract}
The Chi-squared test for contingency tables has good performance when sample sizes are sufficiently large but is not appropriate with small samples. When this condition is violated, Fisher's exact test is often used as it is valid for all sample sizes. However, Fisher's test has been criticised for being overly conservative. Alternatives have been proposed but typically involve more complex computations. In this paper we propose an alternative test based on a geometric projection of the multinomial distribution onto the $\mathrm{n}$-sphere. Each multinomial observation is represented by a single point on this sphere. The angle between two points representing two different multinomial observations can then be compared to the distribution of the angle under the assumption that the realisations come from the same underlying distribution. The null hypothesis distribution is simulated and easy to compute. This new test is compared to the Chi-Squared test and Fisher's Test in terms of both Type I and Type II errors and its potential use in reliability modelling is indicated using a real case study.
\end{abstract}

\section{INTRODUCTION}

Contingency tables are widely used in the areas of safety and reliability modelling when there are several machines, systems or failure types which are possibly spread over different locations. Typically we wish to know whether there are differences between the probability of failure between the different states of two of the different factors such as failure type and location. Examples include (Conte, Rubio, Garcia, \& Cano 2011, Maiti \& Khanzode 2009, Colombo \& Ihm 1988, Schneidewind 1992).

Conte et al. (Conte, Rubio, Garcia, \& Cano 2011) considered a $19 \times 19$ risk-injury contingency table of the accident rates in Spanish companies over an 11 year period to identify separate risk and injury groups. Maiti and Khanzode (Maiti \& Khanzode 2009) investigated a relative risk model using a two way contingency table for fatal roof and side fall accidents at coal mines in India.

In Colombo and Ihm (Colombo \& Ihm 1988) failure rates at nuclear power plants were considered. The failure rates of components were broken down by plant location and reactor system. This led to a contingency table for the numbers of failures which was further complicated by the different exposures at each of the plants. Schneidewind (Schneidewind 1992) was interested in the validation of software metrics. His proposed methodology used contingency tables as an important part of this validation process, counting up the numbers of correct and incorrect classifications of each type.
An example of a contingency table, based on the two factors in Colombo and Ihm (Colombo \& Ihm 1988), is given in Table 1.

\begin{tabular}{|l|lll|}
\hline & \multicolumn{3}{|c|}{ Location } \\
\hline System Type 1 & $n_{1,1}$ & $n_{1,2}$ & $n_{1,3}$ \\
System Type 2 & $n_{2,1}$ & $n_{2,2}$ & $n_{2,3}$ \\
System Type 3 & $n_{3,1}$ & $n_{3,2}$ & $n_{3,3}$ \\
\hline
\end{tabular}

Table 1: An example contingency table with factors Location and System.

In such a contingency table we would typically be interested in whether there were differences in the probabilities or rates of failures which result in the observed numbers of failures $n_{i, j}, i, j=1,2,3$ between the different locations or systems. There are two main statistical tests which could be used to do this; the Chi-Squared Test and Fisher's Exact Test (Pearson 1900, Fisher 1922).

In the Chi-Squared test expected frequencies are calculated for each of the cells in the contingency table $e_{i, j}$ and these are then compared to the observed frequencies to form the Chi-Squared test statistic

$$
\chi^{2}=\sum_{i=1}^{I} \sum_{j=1}^{J} \frac{\left(n_{i, j}-e_{i, j}\right)^{2}}{e_{i, j}^{2}}
$$

which is compared to the $\chi^{2}$ distribution on $(I-$ $1)(J-1)$ degrees of freedom. The Chi-Squared Test is useful when all of the observed frequencies are equal to at least 5 but performance becomes poor 
when at least one of the frequencies is smaller than this (Cochran 1954). It cannot be carried out with multiple observed frequencies of 0 .

Variants to the Chi-Squared Test have been proposed for small sample sizes. Two of the most prominent are the Yates correction and Fisher's Test. In Fisher's Test an exact p-value is calculated based on the assumption that the row and column totals are fixed. While the Fisher Test is exact and so is suitable for small sample sizes it has been criticised for being overly conservative (Liddell 1976). This conservatism stems from the discrete nature of the test statistic and the assumption of fixed marginals. Several other variants have been proposed in the literature but many of these rely on intensive numerical methods and are therefore, for practitioners wishing to test for differences quickly and simply, not always suitable or appropriate.

(Olbrich 1965) proposed a geometric projection of the multinomial distribution onto the n-sphere. Each multinomial observation was represented by a single point on this sphere and the difference between two multinomial realisations of the same degree was represented by the angle between the two points on the sphere. This representation had the nice property that it was variance stabilising. The representation was not commutative, however; if the order of the categories were switched in the multinomial distributions then the points on the sphere and the angle between the points did not remain the same.

In this paper we propose a commutative version of the (Olbrich 1965) representation. We then use this version to define a hypothesis test to test for differences in contingency tables. The test statistic is the angle between the two multinomial realisations. We use the variance stabilising property of the transformation in order to approximate the distribution under the null hypothesis that the two realisations come from the same underlying probability vector. The test does not require intensive numerical methods.

The remainder of the paper is structured as follows. In Section 2 we review the method of (Olbrich 1965), give our proposed commutative adaption and outline the geometric hypothesis test. In Section 3 we investigate the properties of the proposed transformation and in Section 4 we conduct a simulation study to assess the performance of the Geometric Test against the Chi-Squared Test and Fisher's Test for both Type I and Type II errors. Finally, in Section 5 we give an illustrative example of the method and we conclude the paper in Section 6.

\section{GEOMETRIC TRANSFORMATION OF MULTINOMIALS}

\subsection{The Olbrich Transformation}

Consider two realisations from a multinomial distribution in $n$ dimensions, $\left(x_{1}, \ldots, x_{n}\right)$ and $\left(y_{1}, \ldots, y_{n}\right)$.
Let

$x_{T}=\sum_{i=1}^{n} x_{i}, y_{T}=\sum_{i=1}^{n}$,

be the total number of observations from each of these multinomials. Then the proportions of the observations in each of the categories are

$p_{i}=\frac{x_{i}}{x_{T}}, q_{i}=\frac{y_{i}}{y_{T}}$

Olbrich proposed representing the multinomials in spherical co-ordinates as points on an $n$-shphere using these proportions. Denote these points by $\underline{\theta}, \underline{\phi}$ respectively. Then

$$
\begin{aligned}
& \underline{\theta}=\left(\cos ^{-1}\left(\sqrt{p_{1}}\right), \ldots, \cos ^{-1}\left(\sqrt{p_{n}}\right)\right), \\
& \underline{\phi}=\left(\cos ^{-1}\left(\sqrt{q_{1}}\right), \ldots, \cos ^{-1}\left(\sqrt{q_{n}}\right)\right) .
\end{aligned}
$$

We can use such a transformation to calculate the angle between any two multinomial realisations on the $\mathrm{n}$-sphere. This is given in the below result.

Theorem 1. The angle $A_{O}$ between two multinomial realisations $\left(x_{1}, \ldots, x_{n}\right)$ and $\left(y_{1}, \ldots, y_{n}\right)$ with proportions of observations in each category $p_{i}=\frac{x_{i}}{x_{T}}, q_{i}=$ $\frac{y_{i}}{y_{T}}$ is given by

$A_{O}=\cos ^{-1}\left(\sum_{i=1}^{n+1} \sqrt{p_{i} q_{i}\left[\prod_{j=0}^{i-1}\left(1-p_{j}\right)\left(1-q_{j}\right)\right]}\right)$,

where $p_{0}=q_{0}=0$, and $p_{1}=q_{1}=1$.

Proof. We can represent these points in Cartesian coordinates in $(n+1)$ dimensions. That is,

$$
\begin{aligned}
c_{1} & =r \cos \left(\cos ^{-1}\left(\sqrt{p_{1}}\right)\right), \\
c_{2} & =r \cos \left(\cos ^{-1}\left(\sqrt{p_{2}}\right)\right) \sin \left(\cos ^{-1}\left(\sqrt{p_{1}}\right)\right), \\
& \vdots \\
c_{n} & =r \cos \left(\cos ^{-1}\left(\sqrt{p_{n}}\right)\right) \prod_{i=1}^{n-1} \sin \left(\cos ^{-1}\left(\sqrt{p_{i}}\right)\right),
\end{aligned}
$$

$c_{n+1}=r \prod_{i=1}^{n} \sin \left(\cos ^{-1}\left(\sqrt{p_{i}}\right)\right)$.

The radius is $r=\sqrt{\sum_{i=1}^{n+1} c_{i}^{2}}$. This representation can be tidied up, using the fact that $\sin \left(\cos ^{-1}(z)\right)=$ 


$$
\begin{aligned}
\sqrt{1-z^{2}}, \text { to } & \\
c_{1} & =r \sqrt{p_{1}}, \\
c_{2} & =r \sqrt{p_{2}\left(1-p_{1}\right)}, \\
& \vdots \\
c_{n} & =r \sqrt{p_{n} \prod_{i=1}^{n-1}\left(1-p_{i}\right)}, \\
c_{n+1} & =r \sqrt{\prod_{i=1}^{n}\left(1-p_{i}\right) .}
\end{aligned}
$$

We denote the Euclidian co-ordinates for $\underline{\theta}$ as $\left(c_{1}(\underline{\theta}), \ldots, c_{n+1}(\underline{\theta})\right)$ and for $\underline{\phi}$ as $\left(c_{1}(\underline{\phi}), \ldots, c_{n+1}(\underline{\phi})\right)$. Then the angle between these two points is

$$
A_{O}=\cos ^{-1}\left(\frac{\sum_{i=1}^{n+1} c_{i}(\underline{\theta}) c_{i}(\underline{\phi})}{\sqrt{\sum_{i=1}^{n+1} c_{i}(\underline{\theta})^{2} \sum_{i=1}^{n+1} c_{i}(\underline{\phi})^{2}}}\right) \text {. }
$$

This reduces to the result given.

The angle has the nice property that it tends to zero for two multinomial realisations from the same underlying distribution as the sample sizes go to infinity. To see this, consider

$$
\begin{aligned}
\lim _{x_{T}, y_{T} \rightarrow \infty} A & =\cos ^{-1}\left(1-\rho_{n}+\sum_{i=1}^{n} \sqrt{\rho_{i}^{2} \prod_{j=1}^{i-1}\left(1-\rho_{j}\right)^{2}}\right. \\
& =\cos ^{-1}\left(1-\rho_{n}+\sum_{i=1}^{n} \rho_{i} \prod_{j=1}^{i-1}\left(1-\rho_{j}\right)\right) \\
& =\cos ^{-1}(1) \\
& =0 .
\end{aligned}
$$

\subsection{The Commutative Property}

Upon inspection of the angle, $A_{O}$, in Theorem 1 , it is apparent that the choice of label, i.e., $i$, which a category is assigned will influence the resulting value of the angle. That is, if the categories of the multinomial variables are re-ordered, then the angle between them using the Olbrich transformation will be different.

Consider a simple $2 \times 2$ table with the two categories being "blue" and "not blue". Here the test statistic reduces to one of the two following test statistics depending on which category is assigned to be the first category.

$$
\begin{aligned}
a_{O} & =\cos ^{-1}\left(\sqrt{p_{\text {blue }} q_{\text {blue }}}+\left(1-p_{\text {blue }}\right)\left(1-q_{\text {blue }}\right)\right) \\
& \neq \cos ^{-1}\left(\sqrt{\left(1-p_{\text {blue }}\right)\left(1-q_{\text {blue }}\right)}+p_{\text {blue }} q_{\text {blue }}\right) .
\end{aligned}
$$

This non-commutative property is a result of the dependence structure in the probabilities which ensures that they sum to 1 over all categories. As such, if we re-define the probabilities used within the test such that they are conditionally independent then we will have a commutative representation of the angle between two multinomial realisations.

As such, by using probabilities conditionally independent on categories with a lower index value and following the same geometric transformation as in Olbrich's work we can obtain a commutative formula for the angle. This is given in the following theorem. The proof follows the same form as that for Theorem 1. It is left to the reader.

Theorem 2. The angle $A_{G}$ between two multinomial realisations $\left(x_{1}, \ldots, x_{n}\right)$ and $\left(y_{1}, \ldots, y_{n}\right)$ with conditional proportions of observations in each category given that they weren't in any previous categories $\gamma_{i}=\frac{x_{i}}{x_{T_{i}}}, \beta_{i}=\frac{y_{i}}{y_{T_{i}}}$, where $X_{T_{i}}=\sum_{j=i}^{n} x_{j}, Y_{T_{i}}=$ $\sum_{j=i}^{n} y_{j}$, is given by

$$
\begin{aligned}
A_{G} & =\cos ^{-1}\left(\sum_{i=1}^{n+1} \sqrt{\gamma_{i} \beta_{i}\left[\prod_{j=1}^{i-1}\left(1-\gamma_{i}\right)\left(1-\beta_{i}\right)\right]}\right) \\
& =\cos ^{-1}\left(\sum_{i=1}^{n} \sqrt{p_{i} q_{i}}\right),
\end{aligned}
$$

where $p_{0}=q_{0}=0$, and $p_{1}=q_{1}=1$

\subsection{Geometric Hypothesis Test}

Suppose we have two multinomial realisations $\underline{x}$ and $y$ with underlying probability vectors $p$ and $q$ respectively. We wish to test whether the two vectors come from the same underlying multinomial distribution. The hypothesis test will take the form

$H_{0}: \underline{p}=\underline{q}$,

$H_{1}: \quad \underline{p} \neq \underline{q}$.

The test statistics is

$A_{G}=\cos ^{-1}\left(\sqrt{\sum_{i=1}^{n} \hat{p}_{i} \hat{q}_{i}}\right)$,

for observed $\hat{p}_{i}=x_{i} / x_{T}, \hat{q}_{i}=y_{i} / y_{T}$. The p-value is then

$\operatorname{Pr}\left(a \geq A_{G}\right) \approx \frac{1}{N} \sum_{i=1}^{N} I\left(a_{i} \geq A_{G}\right)$,

where $N$ is a large number of simulated values of $A_{G}$ under the assumption that $H_{0}$ is true. To calculate 
$a_{1}, \ldots, a_{N}$ we need to estimate the probability vector under the null hypothesis, $\underline{p}_{0}$. This is

$\underline{\hat{p}}_{0}=\frac{1}{2} \times\left(\underline{\hat{p}}_{1}+\underline{\hat{p}}_{2}\right)$.

Thus we see that to perform the test we carry out a parametric bootstrap (Efron \& Tibshirani 1993) on $p_{0}$.

\section{PROPERTIES OF THE TRANSFORMATION}

We have evaluated the mean and standard deviation of the angle $A_{G}$ resulting from the transformation of one multinomial variable compared with its mean. The aim is to develop some intuition concerning the behaviour of this transformation. We have considered the mean and standard deviation with respect to the underlying probabilities and sample size.

Specifically, we have used Maple 17 software to calculate the mean and standard deviation exactly for multinomial distributions with 3 to 10 categories. In varying the underlying probabilities we used the standard deviation of the probabilities, as this is related to the Euclidean distance of the vector of probabilities from the vector of equal probabilities. Our standard deviations range from 0 to 0.5 . We give two figures below to illustrate the analysis. The overall conclusions made are based on the more comprehensive investigation.

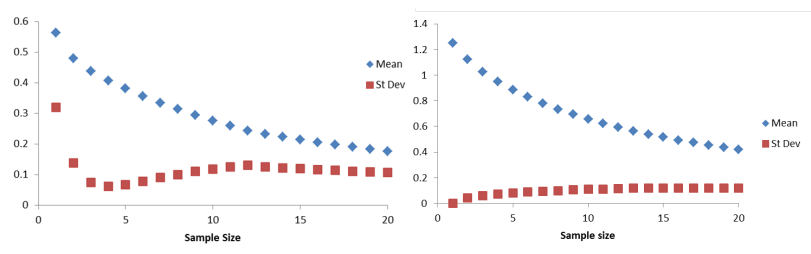

Figure 1: Mean and standard deviation of $A_{G}$ for $m=3$ with standard deviations of 0 (left) and 0.29 (right).

In Figure 1 we see the change in the mean and standard deviation of $A_{G}$ for a 3-dimensional multinomial distribution over different sample sizes. In the left hand plot the standard deviation is 0 and in the right hand plot it is 0.29. In Figure 2 we see the same two plots for a 10 dimensional multinomial distribution.
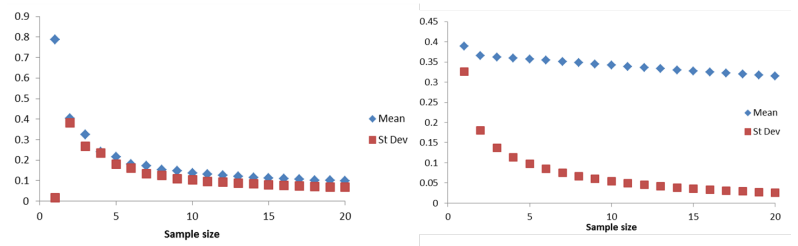

Figure 2: Mean and standard deviation of $A_{G}$ for $m=10$ with standard deviations of 0 (left) and 0.29 (right).

An interesting pattern in the mean of angle from the transformation is that it monotonically decreases as sample size increases. Our study considered 99 different combinations of probabilities, number of categories and sample sizes, from which we are able to
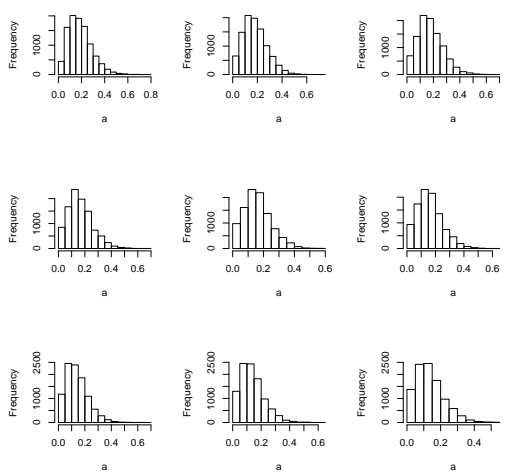

Figure 3: The distribution of $A_{G}$ for 10,000 simulated pairs from the trinomial distribution with the same probability vector.

fit a curve to the relationship between the mean angle, the standard deviation of the probabilities and the number of samples. That is,

$\bar{x}_{A}=1.356-2.8 \times s_{p}+\left(0.8 \times s_{p}-0.34\right) \log (n)$,

where $\bar{x}_{A}$ is the mean of $A_{G}$ and $s_{p}$ is the standard deviation of the probabilities. This excludes the cases with equal probability. The fit of the curve was very good with $R^{2}=87.8 \%$, and it shows that the mean decreases at a rate $o(\log (n))$ and is not affected by the number of categories $m$. The standard deviation of the probabilities influences the mean in two ways; it decreases the mode and increases the rate of decay with respect to sample size.

\section{SIMULATION STUDY}

\subsection{The distribution of $A_{G}$}

We wish to compare two multinomial realisations to test if they come from the same underlying distribution, i.e., if the have the same probability vector.

In Figure 3 we give histograms of the angle $A_{G}$ for the trinomial distribution simulated with the same underlying probabilities for a range of different parameter vectors. In each case 10,000 pairs of points are simulated and there are 50 observations for each simulated point.

We see that in all cases the distribution of $A_{G}$ has a mode which is close to zero and is right skewed. Of course, if the two realisations are the same then the angle between them will be 0 . 


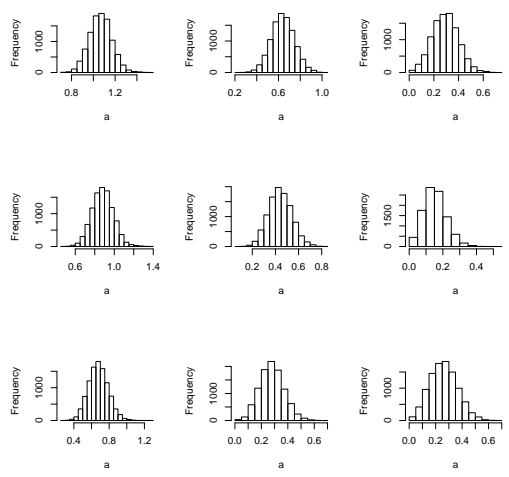

Figure 4: The distribution of $A_{G}$ for 10,000 simulated pairs from the trinomial distribution with different probability vectors.

We can carry out a similar simulation exercise for trinomial realisations which come from distributions with different underlying probability vectors. The resulting histograms are given in Figure 4. Again, each graph is the result of 10,000 simulations.

This time we see that the distributions of $A_{G}$ are generally much more symmetric and have modes which are typically larger than in Figure 3 . This suggests that $A_{G}$ may be a useful statistic within the hypothesis test we have outlined in Section 2.3.

\subsection{Type I and Type II errors}

We will investigate the properties of the test with respect to Type I and Type II errors. All results are based on 1000 samples. The Type I error probabilities are given in Table 3 in the Appendix for two different probability vectors and different sample sizes. The probabilities from the Pearson Chi-squared test are also given.

We see that, for $\underline{p}_{1}$ and $\underline{p}_{2}$ all three tests are performing fairly well, with the proportions of Type I errors being close to 0.05 for all tests over almost all sample sizes. This indicates that, for trinomials in which all of the categories have reasonably large probabilities, all of the tests perform satisfactorally with respect to Type I errors. However, for $\underline{p}_{3}$ in which there are small probabilities of being in some of the categories and hence small numbers of observations in those categories, all three tests have proportions of Type I errors well below 0.05 for a sample size of 5 . The geometric test then performs well from a sample size of 10 whereas the other two tests are still making too few Type I errors up to a sample size of approximately 30 .

The Type II errors are given in Table 4 in the Appendix. In Table 4 probabilities are $p_{1}=$ $(1 / 3,1 / 3,1 / 3), \quad \underline{p}_{2}=(1 / 2,1 / 4,1 / 4)$ and $\underline{p}_{3}=$ $(8 / 10,1 / 10,1 / 10)$.

We see that the Geometric Test outperforms the other two tests for all combinations of probability vectors for sample sizes of 5 and 10. Beyond a sample size of 10 the Fisher Test, in particular, typically outperforms the Geometric Test. However, in this paper we are interested in situations where we have small probabilities and possibly few data and the in this case the Geometric Test give an improvement, albeit small, over the other two tests. When combined with its superior Type I error probabilities for the trinomial distribution with low probability events and this new Geometric Test looks to have some strengths over the two traditional tests.

\section{ILLUSTRATIVE EXAMPLE}

The illustrative example in this section is based on a real case. It has been suitably desensitised for this paper and the numbers used are typical rather than specific values.

A company manufactures machinery which is then sold to operators in the power sector. As part of the contracts for the machinery the manufacturer guarantees specific levels of performance including reliability levels. There are different possible failure types of the machines which at a high level can be classified as Design, Installation, Maintenance and No Fault Found.

The reliability of the machinery depends on how it is operated and how it is maintained. This is not in the control of the manufacturer. It is suspected that there could be systematic differences between different regions as to the reliability of the same equipment as a result of different operating procedures and different operating and maintenance capabilities.

Data have been collected on the numbers of failures by failure type and region and are given in Table 2 .

\begin{tabular}{|c|cccc|}
\hline Type/Region & Europe & N America & Africa & Asia \\
\hline Design & $2(5)$ & $12(12)$ & $7(6)$ & $6(4)$ \\
Installation & $15(11)$ & $20(25)$ & $12(12)$ & $10(9)$ \\
Maintenance & $1(1)$ & $7(3)$ & $0(1)$ & $0(1)$ \\
None Found & $0(1)$ & $4(3)$ & $2(1)$ & $0(1)$ \\
\hline
\end{tabular}

Table 2: Contingency table of the number of failures of specific failure types across different regions.

We wish to test whether there are differences in the failure patterns across the different regions. The expected numbers of failures in each of the cells conditional on fixing the totals across rows and columns are given in brackets in Table 2 . 
Suppose we ask the question: is there a difference in the pattern of failures between Europe and North America? For the Pearson Test $p=0.074$, for Fisher's Test $p=0.090$ and so you cannot reject the null hypothesis that there is no difference in the pattern of failures between the two areas.

If we use the Geometric Test, however, we get a Test Statistic $A_{G}=0.465$ which corresponds to a pvalue of $p=0.049$. Thus we see that the Geometric Test detects the difference between the two areas in this case when the Fisher and Pearson tests do not.

\section{CONCLUSIONS}

In this paper we have considered the analysis on contingency tables for numbers of failures in a reliability context. There will typically be some failure types with low probability and as such small numbers of realised failures in some of the cells in the contingency table. This means that the traditional Chi-Squared Test will be unsuitable and the most common alternative, Fisher's Test, is conservative meaning that statistically significant differences will be missed.

We have considered an alternative to these tests, which we have named the Geometric Test, which represents multinomial observations as points on the nsphere. The test statistic is then the angle between the points and the p-value is approximated by sampling from the distribution under the null hypothesis. This new test out-performed the other two tests with respect to the Type I errors when some categories of the multinomial variable had small probability and with respect to Type II errors for small sample sizes.

There is much work still to be done for this method from both theoretical and applied perspectives. The test needs to be validated on a much larger simulation study and an investigation could be carried out into the theoretical properties of both the test statistic and the test. There is also work to be done in evaluating the performance of the test in more than 3 dimensions and extending the test to more than two simultaneous miultinomial variables. It would also be interesting to benchmark the commutative test against the noncommutative transformation of Olbrich.

From an applied perspective, in order for the method to be useable for reliability practitioners it will be needed to be automated so that large numbers of simulations do not have to be run every time the test is performed.

\section{REFERENCES}

Cochran, W. G. (1954). Some methods for strengthening the common 2 tests. Biometrics 10(4), pp. 417-451.

Colombo, A. \& P. Ihm (1988). A quasi-independence model to estimate failure rates. Reliability Engineering and System Safety 21, 309-318.

Conte, J., E. Rubio, A. Garcia, \& F. Cano (2011). Occupational accidents model based on risk-injury affinity groups. Safety Science 49, 306-314.
Efron, B. \& R. Tibshirani (1993). An Introduction to the Bootstrap. FL: Chapman and Hall/CRC.

Fisher, R. A. (1922). On the interpretation of 2 from contingency tables, and the calculation of p. Journal of the Royal Statistical Society 85(1), pp. 87-94.

Liddell, D. (1976). Practical tests of 22 contingency tables. Journal of the Royal Statistical Society. Series D (The Statistician) 25(4), pp. 295-304.

Maiti, J. \& V. Khanzode (2009). Development of a relative risk model for roof and side fall fatal accidents in underground coal mines in india. Safety Science 47, 1068-1076.

Olbrich, E. (1965). Geometrische deutung der polynomialverteilung und einige folgerungen daraus. Biometrische Zeitschrift 7(2), 96-101.

Pearson, K. (1900). X. on the criterion that a given system of deviations from the probable in the case of a correlated system of variables is such that it can be reasonably supposed to have arisen from random sampling. Philosophical Magazine Series 5 50(302), 157-175.

Schneidewind, N. (1992). Methodology for validation software metrics. IEEE Transactions on Software Engineering 18(5), 410-422. 


\begin{tabular}{cc|ccccccc|}
\hline Test & Probabilities & 5 & 10 & 20 & 30 & 40 & 50 & 100 \\
\hline Geometric & $\underline{p}_{1}$ & 0.061 & 0.062 & 0.051 & 0.055 & 0.046 & 0.049 & 0.043 \\
& $\underline{p}_{2}$ & 0.042 & 0.062 & 0.038 & 0.043 & 0.039 & 0.050 & 0.062 \\
Pearson & $\underline{p}_{3}$ & 0.017 & 0.052 & 0.057 & 0.049 & 0.052 & 0.043 & 0.053 \\
& $\underline{p}_{1}$ & 0.042 & 0.037 & 0.049 & 0.043 & 0.040 & 0.047 & 0.049 \\
& $\underline{p}_{2}$ & 0.031 & 0.047 & 0.059 & 0.035 & 0.051 & 0.049 & 0.048 \\
Fisher & $\underline{p}_{3}$ & 0.005 & 0.011 & 0.037 & 0.040 & 0.040 & 0.056 & 0.043 \\
& $\underline{p}_{1}$ & 0.033 & 0.056 & 0.050 & 0.047 & 0.040 & 0.046 & 0.043 \\
& $\underline{p}_{2}$ & 0.022 & 0.050 & 0.044 & 0.045 & 0.037 & 0.046 & 0.061 \\
& $\underline{p}_{3}$ & 0.006 & 0.011 & 0.032 & 0.031 & 0.045 & 0.039 & 0.052 \\
\hline
\end{tabular}

Table 3: Type I error probabilities for the three tests for various sample sizes.

\begin{tabular}{|cc|ccccccc|}
\hline Test & Probabilities & 5 & 10 & 20 & 30 & 40 & 50 & 100 \\
\hline Geometric & $\underline{p}_{1}, \underline{p}_{2}$ & 0.917 & 0.915 & 0.885 & 0.790 & 0.741 & 0.688 & 0.449 \\
& $\underline{p}_{1}, \underline{p}_{3}$ & 0.771 & 0.569 & 0.278 & 0.078 & 0.023 & 0.004 & 0.000 \\
\multirow{3}{*}{ Pearson } & $\underline{p}_{2}, \underline{p}_{3}$ & 0.893 & 0.783 & 0.643 & 0.448 & 0.292 & 0.171 & 0.011 \\
& $\underline{p}_{1}, \underline{p}_{2}$ & 0.941 & 0.906 & 0.867 & 0.819 & 0.758 & 0.663 & 0.435 \\
& $\underline{p}_{1}, \underline{p}_{3}$ & 0.812 & 0.540 & 0.197 & 0.061 & 0.021 & 0.003 & 0.000 \\
Fisher & $\underline{p}_{2}, \underline{p}_{3}$ & 0.920 & 0.820 & 0.561 & 0.400 & 0.274 & 0.166 & 0.013 \\
& $\underline{p}_{1}, \underline{p}_{2}$ & 0.953 & 0.924 & 0.885 & 0.797 & 0.753 & 0.698 & 0.443 \\
& $\underline{p}_{1}, \underline{p}_{3}$ & 0.821 & 0.576 & 0.226 & 0.070 & 0.020 & 0.003 & 0.000 \\
& $\underline{p}_{2}, \underline{p}_{3}$ & 0.913 & 0.818 & 0.601 & 0.416 & 0.281 & 0.172 & 0.009 \\
\hline
\end{tabular}

Table 4: Type II error probabilities for the three tests for various sample sizes. 